\title{
CIRCULATION AND MIXING
}

\author{
R. KIPPENHAH N \\ Universitäts-Sternwarte, Göttingen, F.R.G.
}

In the preceding lecture Dr Arnett has shown us how exciting the inner life of a star can be when during late stages of evolution higher nuclear reactions take place. But in order to enjoy the variety of different nuclear species formed there stellar material from the deep interior has to be brought up to the surface, where the observers can see it.

When we produce evolutionary sequences of stellar models we simplify in many respects. In particular, we assume that nuclear material stays where it is being formed unless convection brings it to other places.

But there are indications that material from the very interior is occasionally brought to the surface - the technetium stars are probably the most striking examples (Aller, 1961 , p. 159). How can material from a stellar core reach the surface? Maybe during more advanced evolutionary stages stars blow off their envelopes into space and the core material then becomes visible. Maybe convection brings the matter from the deep interior up to the surface of the stars. But it is also possible that other mixing processes become important. The most well known of these is meridional motion in radiative regions of rotating stars. This is a special case of a more common situation where, as a consequence of deviation from spherical symmetry in a star, hydrostatic equilibrium and thermal radiative equilibrium are incompatible, in which case meridional motions always occur. In the first two chapters of this paper we will deal with meridional motions of this type.

Since thermal conduction can be included in the stellar structure equation of radiative transport our considerations also hold in degenerate regions of stars, where conduction is the leading transport mechanism.

But also in the pure spherically symmetric case, where hydrostatic equilibrium and thermal radiative equilibrium can be fulfilled simultaneously meridional motion can occur. If in such a case a nonspherical perturbation is applied which is so slow that hydrostatic equilibrium is still a good approximation but which perturbs the thermal part of the stellar structure equations, then again circulation will occur which either enhances the perturbation or diminishes it. In the first case one has a secular instability which can give rise to meridional circulation. In Section 3 we give examples for this case of circulation.

\section{Meridional Circulation}

\subsection{EDDINGTON-VOGT CIRCULATION}

In the following we deal first with slowly rotating stars. The ratio $\chi_{r}$ of centrifugal to gravitational acceleration in the equatorial plane at distance $r$ from the center is 
therefore assumed to be small. Then meridional motions occur in radiative regions with velocities of the order of

(see A1) with

$$
v_{\mathrm{EV}} \approx \frac{\nabla_{\mathrm{ad}}}{\delta\left(\nabla_{\mathrm{ad}}-\nabla\right)} \frac{L_{r} \chi_{r}}{M_{r}} \frac{3}{4 \pi r G \varrho}
$$

$$
\nabla=\frac{\mathrm{d} \ln T}{\mathrm{~d} \ln P}, \quad \nabla_{\mathrm{ad}}=\left(\frac{\mathrm{d} \ln T}{\mathrm{~d} \ln P}\right)_{\mathrm{ad}}, \quad \delta=-\left(\frac{\partial \ln \varrho}{\partial \ln T}\right)_{P}
$$

while $M_{r}, L_{r}, r, G, \varrho$ have the usual meaning. $\delta$ has to be determined from the equation of state. For the perfect gas one has $\delta=1$. With increasing degeneracy $\delta \rightarrow 0$ which is of importance if Equation (1) is applied to degenerate regions in a star.

The existence of meridional circulation in radiative regions has been postulated independently by Eddington (1925) and by Vogt (1925), by discussing von Zeipel's theorem. The first numerical estimate was given by Sweet (1950); therefore this type of motion is sometimes called Eddington-Sweet circulation.

If we take the mean over the whole star Equation (1) yields

$$
\overline{v_{\mathrm{EV}}} \approx \frac{1}{\overline{\delta\left(\nabla_{\mathrm{ad}}-\nabla\right)}} M_{\bar{g}} \overline{\bar{\gamma}_{r}}
$$

where bars indicate mean values over the star, while $g=G M / R^{2}$ is the surface gravity at the equator. In Equation (3) we have neglected dimensionless factors of the order one. If we introduce the Kelvin-Helmholtz time scale $\tau_{\mathrm{KH}} \approx E_{G} / L$ of the star where $E_{G} \approx G M^{2} / R$ is the absolute value of the gravitational energy of the star we find

$$
\overline{v_{\mathrm{EV}}} \approx \frac{1}{\delta\left(\nabla_{\mathrm{ad}}-\nabla\right)} \tau_{\mathrm{KH}}^{R} \overline{\bar{\chi}_{r}} .
$$

Connected with this mean velocity there is a characteristic time scale given by

$$
\tau_{\mathrm{EV}} \approx \frac{R}{\bar{v}_{\mathrm{EV}}} \approx \frac{\overline{\delta\left(\nabla_{\mathrm{ad}}-\nabla\right)}}{\bar{\chi}_{r}} \tau_{\mathrm{KH}} .
$$

The Eddington-Vogt circulation follows from the fact that hydrostatic equilibrium in a rotating star and thermal radiative equilibrium cannot be fulfilled simultaneously. Formally this can be seen if one writes down the equations for rotational perturbations. One then finds that the system is overdetermined until a new variable, the velocity of meridional motion is introduced (Kippenhahn, 1963).

The estimates for $v_{\mathrm{EV}}$, given here, are derived from a first order perturbation theory which holds only for small values of $\chi_{r}$. This is sufficient for most applications, since $\chi_{r}$ is small in the interior of uniformly rotating stars, even if they rotate with break up velocity. In the case of differential rotation with high angular velocities in the interior of the stars, our estimates are no longer valid. But even in the case for which $\chi_{r} \approx 1$ throughout the star, they may give the order of magnitude of the circulation occurring. 


\section{2. $\mu$-Circulation}

Let us assume a chemically homogeneous region in a star mainly consisting of hydrogen and let us put a blob of pure helium somewhere in that region outside the center. If at the beginning the blob is not in hydrostatic equilibrium it first will expand or contract until the pressure inside is the same as outside. For any quantity $y$ we define by $D y$ the difference between the value of $y$ in the blob and the value of $y$ in the immediate surroundings. Hydrostatic adjustment then means that for the pressure $P$ we have $D P=0$. Then in general the density will not yet be the same in the blob as outside and the blob will rise or sink until there is no buoyancy force, i.e. until $D \varrho=0$. For the sake of simplicity we assume a perfect gas inside and outside the blob. Then as a consequence of the different mean molecular weights $\mu$ we have $D \mu>0$ and therefore $D T>0$. This means that the matter in the blob will be hotter than the surroundings. Therefore there is no thermal equilibrium. The blob will now try to adjust its temperature to the neighbourhood. It therefore will cool off, its density will increase and the blob will sink (Figure 1), with a velocity which is controlled by the thermal adjustment time. It is given by (see A2):

$$
v_{\mu} \approx \frac{\varphi H_{P}}{\delta\left(\nabla_{\mathrm{ad}}-\nabla\right)} \tau_{\mathrm{KH}}^{*} \quad \mu,
$$

where $\varphi=(\partial \ln \varrho / \partial \ln \mu)_{P, T}$ follows from the equation of state. For the perfect gas $\varphi=1 . H_{P}$ is the pressure scale height while $\tau_{\mathrm{KH}}^{*}$ is the thermal adjustment time scale (Kelvin-Helmholtz time scale) of the blob. It is given by (see for instance Kippenhahn, 1969)

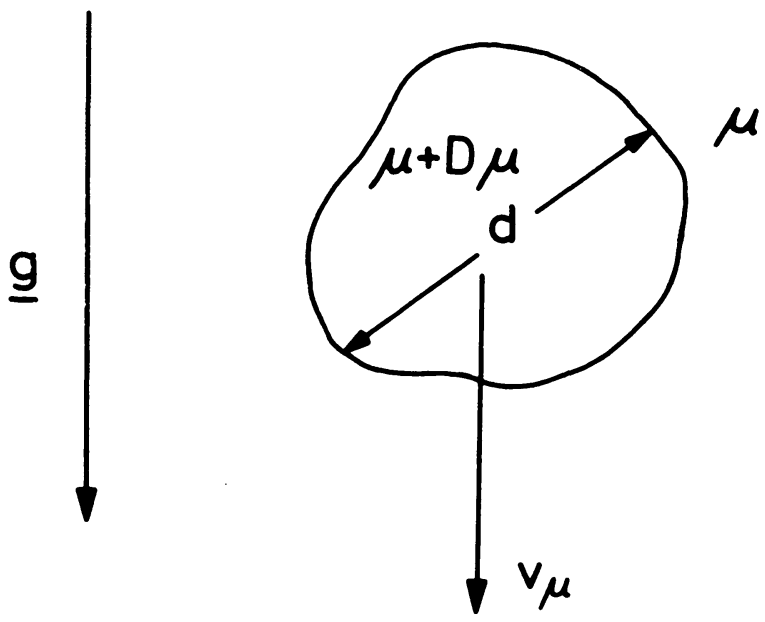

Fig. 1. A blob of molecular weight $\mu+D \mu(D \mu>0)$ in surroundings with molecular weight $\mu$ in hydrostatic equilibrium undergoes a slow downward motion which is controlled by the thermal adjustment of the blob. 


$$
\tau_{\mathrm{KH}}^{*} \approx \frac{3 c_{P} \psi \varrho^{2} \zeta d^{2}}{8 a c T^{3}}
$$

where $d$ is a measure for the size of the blob while $\zeta$ is a dimensionless factor which depends on the geometry. If $V$ and $O$ are volume and surface of the blob respectively, then one has $\zeta=V / O d$.

The time of thermal adjustment as it is defined in Equation (7) increases with the size of the blob. For blobs with a size comparable or even bigger than $H_{P}$, for $\varkappa, \varrho, T$ in Equation (7) mean values over the blob have to be taken. If the size becomes comparable with the radius of the star then $\tau_{\mathbf{K H}}^{*}$ approaches the Kelvin-Helmholtz time scale of the star: $\tau_{\mathbf{K H}}^{*} \rightarrow \tau_{\mathbf{K H}}$.

\subsection{Magnetic BuOYANCY}

We now consider a magnetic blob, that is a subregion in a star which is filled with magnetic field but whose magnetic field lines are entirely in that blob; i.e. no field lines connect the inside with the outside. Finzi and Wolf (1968) used the word 'magnetic tangle' for such an element. Again if there is hydrostatic equilibrium between the magnetic tangle and the surroundings, we have $D P=0, D \varrho=0$. But in the blob the total pressure $P$ consists of the gas pressure $P_{G}$ and the magnetic pressure $P_{M}$. Therefore and if we again assume the perfect gas equation of state we find $D T<0$. This time the blob is cooler than its surroundings and it will try to adjust itself thermally and will rise. The upward velocity is this time given by (see A3)

$$
v_{M} \approx \frac{H_{P}}{\delta\left(\nabla_{\mathrm{ad}}-\nabla\right)} \tau_{\mathrm{KH}}^{*} P_{G}, \quad\left(P_{M}=-D P_{G}\right)
$$

$c_{P}, \varkappa, a, c$ again have the usual meaning, while $\alpha=(\partial \ln \varrho / \partial \ln P)_{T}$.

\subsection{NON-CONSERVATIVE ANGULAR VELOCITY DISTRIBUTION}

There is another, more complicated, case in which hydrostatic equilibrium and thermal radiative equilibrium are not compatible. Let us assume that in a rotating star the angular distribution $\omega$ is not a function of the distance $s$ from the axis of rotation only, but varies on coaxial cylinders $s=$ const. If $z$ is the coordinate in the direction of the axis of rotation, we have in this case $\partial \omega / \partial z \neq 0$. Then the centrifugal acceleration

$$
\mathbf{c}=\omega^{2} \mathbf{s}
$$

is a non-conservative vector field; i.e. $\nabla \times \mathbf{c} \neq 0$. The equation for hydrostatic equilibrium in the steady state is

$$
{ }_{0}^{1} \nabla P+\nabla \phi=\mathbf{c}
$$


where $\phi$ is the gravitational potential. If the temperature were constant on surfaces of equal pressure then the density would also be constant there and $\nabla P / \varrho$ would be a conservative vector field

$$
\nabla \times\left({ }_{\varrho}^{1} \nabla P\right)=0
$$

then the left hand side of Equation (10) would be conservative, but the right hand side would not. Therefore hydrostatic equilibrium demands that the temperature is not constant on pressure surfaces. Therefore a special temperature distribution is necessary to maintain hydrostatic equilibrium. This temperature distribution is not that of thermal radiative equilibrium and consequently meridional motions ensue. This is a type of motion which comes from the Goldreich-Schubert-Fricke instability (Goldreich and Schubert, 1967; Fricke, 1968) of all angular velocity distributions with $\partial \omega / \partial z \neq 0$.

\subsection{LiMIT TOWARDS SMALL LENGTH SCALES}

In the formulae (6) and (8) the velocity of the blob is higher for smaller blobs. This is due to the shorter time scale for thermal adjustment. For smaller scales these formulae are no longer valid since other effects become important, for instance friction. The smaller the blob, the more important the friction. A rough estimate for the lower limit of sizes of blobs can be obtained if one assumes that for the smallest sizes the Stokes velocity becomes comparable with the blob velocity. One then finds that for the order of magnitude of the smallest blobs (see A4)
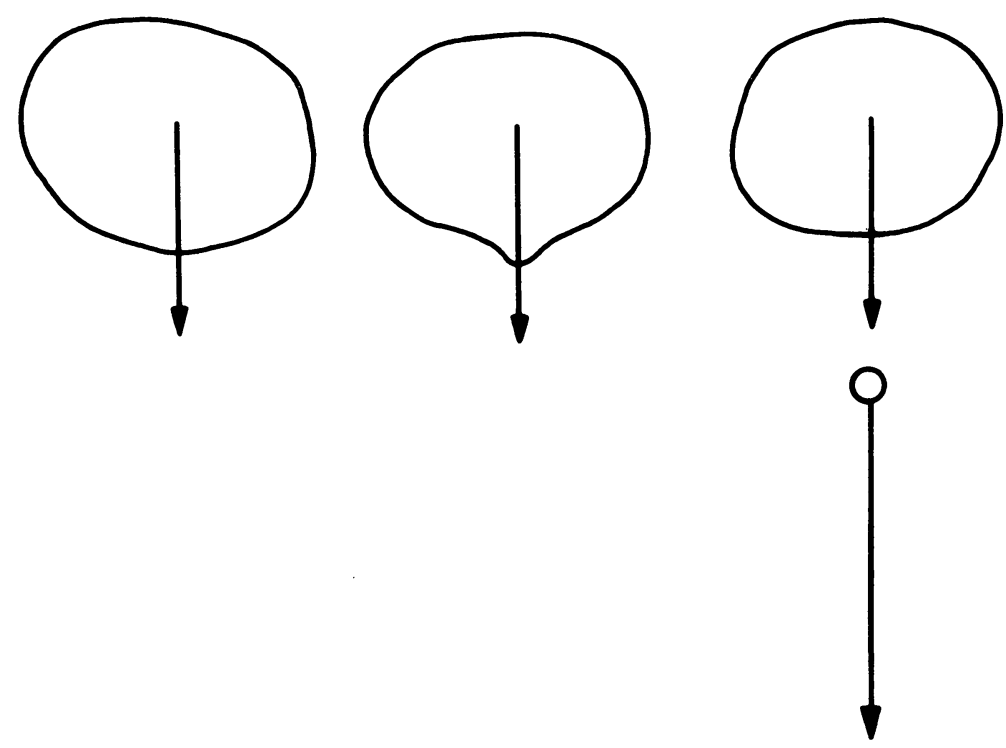

Fig. 2. Since smaller blobs sink faster there is a tendency of fragmentation. 


$$
d \approx \sqrt{\frac{v_{R} c}{g_{r}}},
$$

where $v_{R}$ is the local kinematic radiative viscosity and $g_{r}$ the local gravitational acceleration. As a consequence, big blobs undergo fragmentation during their motions (see Figure 2).

Sometimes when viscosity is small the velocity for small blobs becomes of the order of the velocity of sound before friction effects set in. Then the assumption used in deriving the velocities is no longer valid.

For magnetic tangles $d$ cannot be too small, in order to avoid a decay of the magnetic field before the element has moved over an appreciable distance.

In the case of non-conservative angular velocity distribution discussed in subSection 1.4 the motions which occur will also be small scale motions, since these modes can transport energy more effectively than large scale motions. Indeed in a normal star one should expect sizes of the order of $1 \mathrm{~km}$. Some simple estimates of velocities and time scales of this type of motion have been given by Kippenhahn (1969).

\section{Applications}

\subsection{Mixing in CHEMiCAlly hOMOgENEOUS REgions}

Since on the main sequence the nuclear time scale is roughly 100 times longer than the Kelvin-Helmholtz time-scale with $\delta \approx 1, \nabla_{\mathrm{ad}}-\nabla \approx 0.15$ one can estimate that $\tau_{\mathrm{EV}}$ will be comparable with the time scale $\tau_{\text {nucl }}$ of nuclear evolution if $\bar{\chi}_{r}>0.015$. Indeed for main sequence stars earlier than F5 the Eddington-Vogt time scale is shorter than the main sequence life-time. This has already been found by Sweet (1950). But there is observational evidence that even early type main sequence stars undergo unmixed evolution. For mixed evolution the stars would move to the upper left of the HR diagram while consuming their hydrogen but we are certain that they normally become red giants which is typical for unmixed evolution. Mestel (1953) found the way out of this difficulty. That there is no mixing of hydrogen and helium during the main sequence stage - not even for the fastest rotators - is due to the fact that with the same time-scale a gradient of molecular weight $\mu$ is being built up which prevents circulation, as we will later see. But the regions in these stars which remain chemically homogenous will indeed be mixed by the circulation. The flow pattern is rather complicated. Let us start say with rigid body rotation. For this type of motion the Eddington-Vogt circulation is a large scale motion rising at the poles and sinking at the equator. But since friction can be neglected each mass element conserves its angular momentum. Therefore after a while there will be a deviation from solid body rotation and $\omega$ will not be constant on cylinders $s=$ const. any more. As a consequence small scale motions will occur since the centrifugal acceleration is not conservative. Therefore a new, rather complicated $\omega$-distribution will be established. The Eddington-Vogt circulation of this complicated circulation pattern will be even more complicated. One therefore should expect a 
kind of irregular small scale motion comparable with convection but smaller in scale and much slower. Probably the formula (1) will still give a good estimate for that type of small scale motion which sometimes is called random circulation. This circulation will mix angular momentum throughout the star. Although we do not know what the mean properties of that randomly fluctuating circulation field will be, we would expect that large differences in angular velocity would be smeared out although solid body rotation is not a solution.

Random circulation would also mix chemical elements as long as they do not effect the molecular weight considerably. This might be of some importance for the distribution of lithium through the envelopes of the stars. Recently Paczyński (1973) has suggested that the anomalous ratio of carbon to nitrogen in some early type stars can be explained by mixing. In his picture carbon is being transformed into nitrogen above the convective core in a region where helium is not enriched and therefore the molecular weight is practically constant. Random circulation could bring carbon from the outer layers into that region in which it is destroyed and consequently a large fraction of the carbon in the envelope can be transformed into nitrogen. Demarque and McClure (1973) have given arguments which favour this hypothesis.

Also in degenerate cores of evolved stars circulation might be important. Let us for instance take a degenerate carbon core in an evolved star before the onset of carbon burning. If this core were strictly isothermal, which means energy is neither created nor swallowed in the core, then we would have $L_{r}=0$ for the core and $v_{\mathrm{EV}}$ would therefore vanish. But while the core mass is increasing due to helium burning at its surface, the core contracts and contraction energy is released, giving rise to a non-vanishing $L_{r}$ and therefore a non-vanishing circulation. Neutrino-processes on the other hand take energy away and therefore energy has to be transported from one part of the core to the other, giving rise again to a non-vanishing $L_{r}$ and therefore to circulation. It has been shown by Kippenhahn and Möllenhoff (1974) that the contraction energy released and the energy carried away by neutrinos in a rotating core cannot compensate each other. Therefore there will always be a conductive flux through the core, giving rise to a non-vanishing Eddington-Vogt circulation. By extrapolating the expressions for the circulation velocity derived for slow rotation to the case of $\chi_{r} \approx 1$ Kippenhahn and Möllenhoff (1974) have estimated that in rapidly spinning cores circulation can redistribute angular momentum within the time-scale of evolution. But this is only the case if the cores are spinning so rapidly that they are very flat and that $\chi_{r}$ is of the order 1 . This might be of importance if one discusses the effect of rotation on the conditions for the onset of carbon burning (Sackmann and Weidemann, 1972).

Kippenhahn and Möllenhoff (1974) have also estimated the time-scale for mixing in rapidly rotating white dwarfs. Although white dwarfs can be roughly described as isothermal bodies due to their cooling, $L_{r}$ does not vanish and if $\bar{\chi}_{r}=1$ then the mixing time-scale is comparable with the cooling time. This might be of impor tance for the discussion of rapidly rotating white dwarfs with masses far above the 
Chandrasekhar limit. The random circulation which then would occur would redistribute the angular velocity in a way similar to friction.

\section{2. $\mu$-BARRIERS}

If, say, a helium core in a hydrogen rich envelope has been formed, then, in a transition region, the mean molecular weight $\mu$ increases inwards. If the Eddington-Vogt circulation tries to build up a circulation which, say, rises at the pole and sinks at the equator, then helium rich material is brought up along the polar axis while hydrogen rich material is brought inwards at the equator. This creates a situation where $\mu$-currents try to reestablish spherical symmetry of the $\mu$-distribution and act against the Eddington-Vogt circulation. For a rough estimate we describe the situation given in Figure 3 by a blob of the diameter $d \approx r$ where $r$ is the core radius, we assume
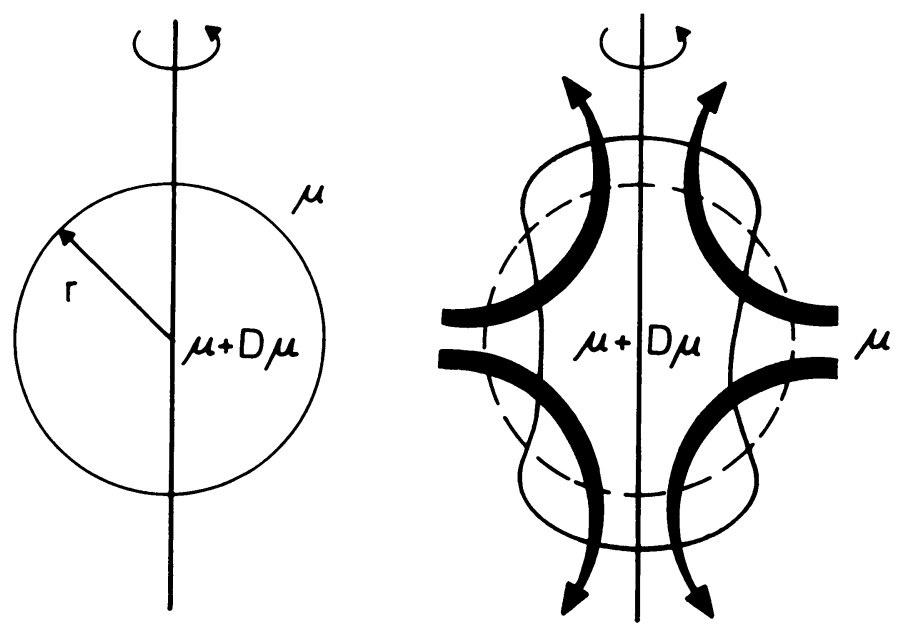

Fig. 3. In the case of a core of molecular weight $\mu+D \mu$ surrounded by an envelope of molecular weight $\mu$ meridional motions (black arrows) produce a non-spherical $\mu$-distribution. Then $\mu$-currents occur which try to reestablish spherical symmetry.

$\tau_{\mathrm{KH}}^{*} \approx \tau_{\mathrm{KH}}$ and we consider the matter in the equatorial plane as the 'surroundings' of the blob. In this very crude picture we can determine $v_{\mu}$ from Equation (6). No mixing will occur if $v_{\mathrm{EV}}<v_{\mu}$. This gives (with $\varphi=1$ ):

$$
\chi_{r}<\left(\frac{2 H_{P}}{r}\right) \frac{|D \mu|}{\mu} \text {. }
$$

The factor $2 H_{P} / r$ may be of the order 1 , and since we have pure helium in the blob and a hydrogen rich mixture $\left(X_{\mathbf{H}}=0.7, X_{\mathrm{He}}=0.3\right)$ outside, $D \mu / \mu$ is roughly 0.74 . Thus one finds that there will be no mixing as long as $\chi_{r}<0.74$. Meridional circulation will occur in the homogenous regions outside and inside the transition region. The region of variable molecular weight acts as a barrier, it prevents the Eddington-Vogt circulation from penetrating and mixing. For higher nuclear burning the differences in 
molecular weight which are being built up become smaller. For the transition from helium to a carbon-oxygen mixture one already has $D \mu / \mu \approx 0.27$. Therefore penetration of these $\mu$ barriers is more probable for higher nuclear burning. But even then rotation would have to be rather fast.

\subsection{FORMATION OF $\mu$-BARRIERS}

In the foregoing we have discussed the problem of mixing through a $\mu$-barrier which is already there - but in the star $\mu$-barriers are being built up from homogenous regions by nuclear burning. There is no $\mu$-barrier at the beginning. The question is whether mixing is strong enough to prevent the formation of a $\mu$-barrier. This problem has first been investigated by Mestel (1957). At the beginning there is only EddingtonVogt circulation. If a blob rises along the polar axis is still has the original chemical composition. After the time $\tau_{E V}$ it might sink towards the equatorial plane (Figure 4 ).

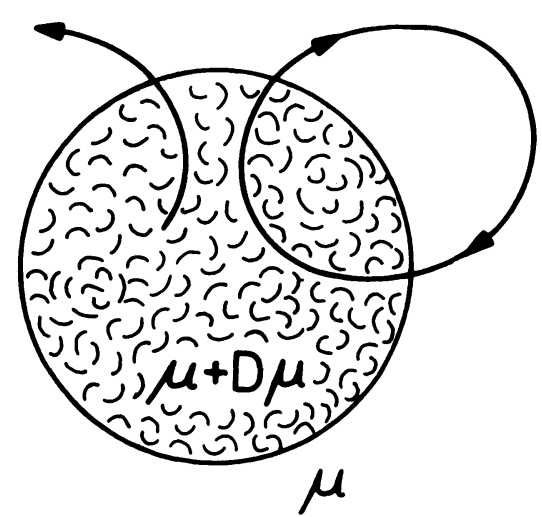

Fig. 4. Meridional circulation in the central region of a star with convective core. Since the molecular weight in the core increases with time the molecular weight varies along the stream lines of the meridional velocity field. Again a non-spherical $\mu$-distribution occurs which gives arise to $\mu$-currents.

At the same time new material is coming up at the pole, enriched in ashes. If we define the rising material as the blob and the sinking material as its surroundings then we have $\left(\tau_{\mathrm{KH}}^{*} \approx \tau_{\mathrm{KH}}\right)$

$$
v_{\mu} \approx \underset{\phi\left(\nabla_{\mathrm{ad}}-\nabla\right) \tau_{\mathrm{KH}} \quad \mu}{\mu},
$$

where again $D \mu$ is the difference in molecular weight between the blob and the surroundings. $D \mu$ has now to be distinguished from $\Delta \mu$ which is the difference of molecular weight before the onset of nuclear burning and after nuclear burning has been completed. One has

$$
D \mu \approx \frac{\partial \mu}{\partial t} \tau_{\mathrm{EV}}, \quad \frac{1 \partial \mu}{\mu t}=\tau_{\tau_{\text {nucl }}}^{1} \frac{\Delta \mu}{\mu}
$$


where $\tau_{\text {nucl }}$ is the time scale of nuclear burning. Therefore $D \mu=\tau_{\mathrm{EV}} \Delta \mu / \tau_{\text {nucl }}$. With this expression for $D \mu$ Equation (6) gives an estimate for the velocity with which the $\mu$-currents try to reestablish spherical symmetry of the $\mu$-distribution. No mixing will occur if $v_{\mathrm{EV}}<v_{\mu}$. In order to estimate $v_{\mathrm{EV}}$ in the neighbourhood of the interface we find from Equation (1)

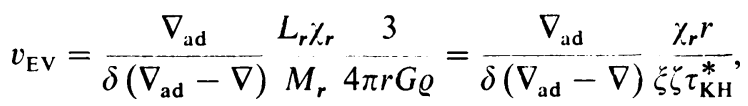

where we have introduced the dimensionless quantities $\xi$ and $\zeta$ by

$$
\zeta=4 \pi r^{3} \varrho / 3 M_{r}, \quad \zeta=G M_{r}^{2} / r L_{r} \tau_{\mathrm{KH}}^{*} .
$$

For the characteristic time connected with these motions near the interface we define

$$
\tau_{\mathrm{EV}}=\frac{r}{v_{\mathrm{EV}}}=\frac{\delta\left(\nabla_{\mathrm{ad}}-\nabla\right)}{\nabla_{\mathrm{ad}}} \frac{\xi \tau_{\mathrm{KH}}^{*}}{\chi_{\mathrm{r}}} .
$$

Here occurs a difficulty since $\nabla_{\mathrm{ad}}-\nabla$ vanishes at the interface between a convective core and a radiative envelope and Equation (16) gives infinite velocities. Mestel (1953) has clarified the situation. In our problem it is sufficient to make clear that for $\nabla_{\mathrm{ad}}-\nabla$ in Equation (17) a value has to be taken which is typical for the whole area through which matter coming out from the core is moving. From Equations (6), (16) and (17) we now obtain the condition that no mixing will occur if

$$
\chi_{r}^{2}<\frac{H_{P}}{r} \varphi \xi^{2} \zeta^{2} \tau_{\text {nucl }}^{\tau_{\mathrm{KH}}^{*}} \frac{\delta\left(\nabla_{\mathrm{ad}}-\nabla\right)}{\nabla_{\mathrm{ad}}^{2}} \mu \mu
$$

For any given stellar model the right hand side of Equation (18) can be determined and critical values of $\chi_{r}$ can be obtained. In order to give a rough idea we use some typical values and obtain from Equation (18) $\chi_{r}<0.1(\Delta \mu / \mu)^{1 / 2}$ and conclude that between a helium core and a hydrogen-helium envelope $(\Delta \mu / \mu=0.74)$ no mixing will occur as long as $\chi_{r}<0.09$ at the interface. For a carbon oxygen core in a helium envelope $(\Delta \mu / \mu=0.27)$ one obtains $\chi_{r}<0.05$. As an example we can take an early type main sequence star in uniform rotation which is fully rotating - that is rotating at an angular velocity so high that centrifugal force and gravitational force cancel each other at the equator. The value of $\chi_{r}$ at the interface between convective core and envelope is then about $1 / 30$ and no mixing will take place (Mestel, 1953). It should be kept in mind that in deriving and in using the condition (18) many dimensionless factors have been dropped in order to give some very simple estimates. For any given stellar model the criterion (18) can be derived in a more careful way following our procedure.

It seems surprising that main sequence stars are not too far from mixing and they might even mix if the angular velocity were to increase inwards sufficiently. On the other hand there is the well established fact that main sequence stars evolve to the right in the HR diagram which is typical for an unmixed evolution. Probably Equation (18) still exaggerates the danger of mixing. Indeed, in deriving this equation it was 
assumed that the Eddington-Vogt circulation is a large scale motion, where the characteristic lengths are given by the radius of the star. But as we have already seen Eddington-Vogt circulation by itself, starting from rigid body rotation, would very soon produce random circulation. Therefore, if in a main sequence star, helium enriched material would be brought out by circulation, say, at the polar region, then it would not move with the velocity $v_{\mathrm{EV}}$ but a random walk process would determine the diffusion of helium into the outer layers. Thus, the velocity by which helium is brought outwards is slower, and from this one might expect that even values of $\chi_{r}$ which are higher than the limit given by Equation (18) would still not cause mixing.

As we have seen in the case of the already fully developed $\mu$-barrier for higher nuclear reactions the $\mu$-effect becomes less and less important. For highly developed stars it therefore depends on the angular velocity of the cores whether mixing can take place or not. Unfortunately, there is practically no information whether the material in the very interior of a star conserves its angular momentum, whether the core which contracts during the evolution will rotate faster and faster or whether angular momentum is being exchanged between core and envelope. Since $\mu$-barriers cannot be penetrated by circulation the only mechanism which brings angular momentum across the $\mu$-barriers are magnetic fields with the appropriate topology. Fricke and Kippenhahn (1972) have used the angular velocities of pulsars in order to estimate that the cores of evolved stars at least rotate faster than the envelopes. But it is not clear whether the cores are really spinning rapidly.

\subsection{Magnetic Mixing}

If, as suggested in the last section, magnetic fields would transfer angular momentum from the cores to the envelopes, then rather strong magnetic fields are necessary, even if the initial poloidal field were small. Differential rotation between core and envelope would form a fairly strong toroidal field in the transition region and the question arises whether magnetic tangles are being formed, which would rise into the envelope. In the rotating convective cores of the stars one might expect dynamo effects similar to those discussed by Parker (1970), Krause and Rädler (1971), Köhler (1973) in connection with the solar cycle. One therefore can ask whether magnetic fields created in the very interior of the stars cannot mix the stars and maybe even bring nuclear material from the very interior to the surface. Gurm and Wentzel (1967) and Finzi and Wolf (1968) have discussed the effect of magnetic mixing. Gurm and Wentzel found that the velocity of the rising blobs should be sufficient to bring material from the core to the surface of a main sequence star in a time shorter than the time of nuclear evolution if the ratio of magnetic to gas pressure exceeds the value $10^{-4}$ (in order to get sufficient buoyancy) and if the diameter of the tangles is smaller than $10^{10} \mathrm{~cm}$ (in order to get a sufficiently short time of thermal adjustment). Finzi and Wolf even suggest that blue stragglers observed in the HR diagrams of globular clusters might be stars which undergo mixed evolution due to magnetic mixing.

The problem of magnetic fields in the very interior is also of importance for the 
problem of the solar neutrinos. Magnetic fields can reduce the observable solar neutrino flux considerably if they are strong enough. On the other hand strong magnetic fields in the very interior of the Sun would rise to the surface due to magnetic buoyancy. One has to keep in mind that the enrichment in helium in the central region of the Sun provides $\mu$-currents which act against magnetic mixing. Equating the two expressions (6) for $\mu$-currents and (8) for the velocity of magnetic blobs indicates that the magnetic fields would stay in the central region as long as

$$
\varphi \frac{D \mu}{\mu}>\frac{P_{M}}{P_{G}} .
$$

For $\mu$ and $\mu+D \mu$ we take the values for the envelope and the center of the present Sun and find $D \mu / \mu=0.3$. With the central pressure of $2.2 \times 10^{17}$ we find that the magnetic fields up to $1.3 \times 10^{9} \mathrm{G}$ can be kept in the very interior of the Sun by the heavier molecular weight there.

If the magnetic field in the interior is stronger than that given in inequality (19), then tangles containing heavier material can move upwards bringing the heavier material into the outer regions. But this transport mechanism is not very effective. If at the beginning the magnetic buoyancy exceeds the extra weight of the heavier material, then while the tangle is rising it is expanding, the magnetic pressure is being reduced in such a way that $P_{M} / P$ is getting smaller. After a finite path the tangle will come to an equilibrium position where the reduced magnetic buoyancy just balances the extra weight of the heavier material (see A5). In this position the tangle will not only be in hydrostatic but also in thermal equilibrium with its surroundings. Then with the decay time of the magnetic field the blob will sink again.

\section{Nonspherical Instabilities in Circularly Symmetric Stars}

Up to now we have always discussed cases where the star shows some deviation from spherical symmetry and where hydrostatic equilibrium and thermal radiative equilibrium cannot be fulfilled at the same time. Now we discuss spherically symmetric stars where indeed both equilibria can be fulfilled but where small deviations from symmetry will grow, giving rise to meridional motion which might mix the star. In accordance to the foregoing procedure we assume perturbations which are sufficiently slow so that hydrostatic equilibrium is fulfilled and the instability is of purely thermal origin. This type of instability is normally called secular instability. We do not mean that the fully developed motions which occurs from this type of instability must be slow and that hydrostatic equilibrium must always be fulfilled. But since we are only discussing the onset of this instability, we can work with hydrostatic equilibrium, which simplifies the problem extremely and which also gives us the possibility to use some of the formalisms given in the preceding chapters.

\subsection{CARBon Fingers}

If neutrino losses are taken into account the helium flash of a $1.3 M_{\odot}$ star occurs in a 
shell (Thomas, 1967). Therefore the carbon is being formed outside the center while the central region still contains the helium with the original low carbon content. After the helium flash helium burning continues to take place in a shell. Therefore a configuration occurs which is given in Figure 5. Consequently, we now discuss the case of a layer enriched in helium, which is above a helium layer as indicated in Figure 6. If one makes a small perturbation of the interfaces, then a drop of carbon which is hanging into the carbon region will, after reestablishing hydrostatic equilibrium, be hotter than the helium at the same level and we have the problem of $\mu$-motion as discussed in sub-Section 1.2. Therefore the carbon enriched blob will sink even further and one has a situation similar to the salt finger experiment. The carbon will mix with the helium region below. From Thomas's models one obtains the following numerical values (in cgs units):

$$
\begin{aligned}
& H_{P}=6 \times 10^{6}, \quad d=24, \quad \tau_{\mathrm{KH}}^{*}=1.5 \\
& \frac{1 \mathrm{~d} \mu}{\mu \mathrm{d} t} \approx 10^{-5},
\end{aligned}
$$

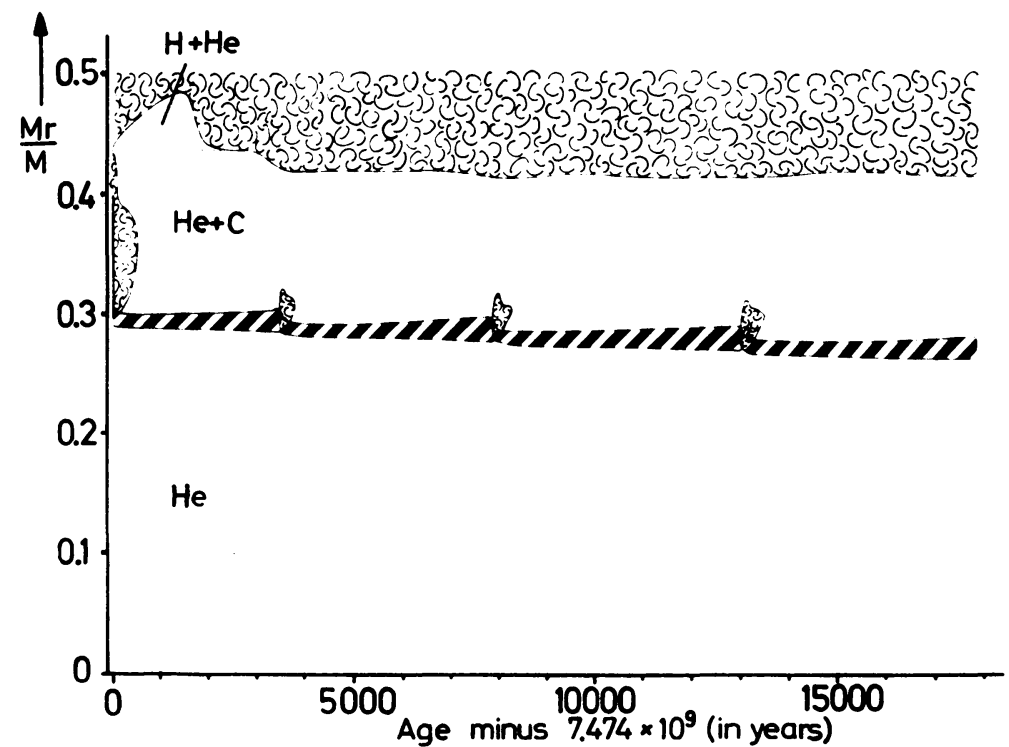

Fig. 5. Helium burning in a shell after the helium flash of a star of $1.3 M_{\odot}$ (Thomas, 1967). The cloudy regions indicate convection. The hatched region indicates shell burning. The regular variations of the shell including the small, short living convective zones just above the shell are due to thermal pulses. In the shell a layer is being built up where the molecular weight is higher than in the layers below.

where $d$ again is determined by Equation (12). After the first second the velocity of the carbon enriched blobs will therefore be some $10 \mathrm{~cm} \mathrm{~s}^{-1}$. Elements which start later will have higher velocity and will pass the earlier ones. This mixing problem has not yet been treated in detail. Thomas (1967) has assumed for his calculations complete mixing of the carbon enriched shell with the interior. 


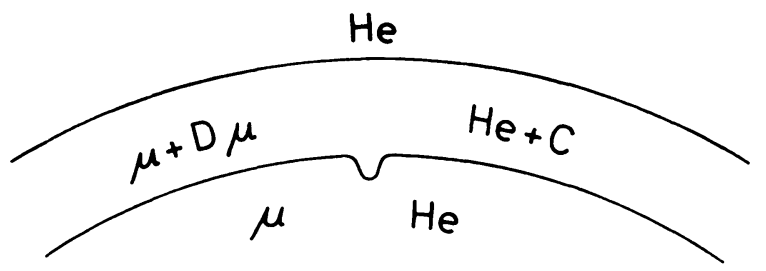

Fig. 6. The situation described in Figure 5 now in a simplified picture. The carbon enriched shell is secularly unstable and carbon fingers will move downwards.

\subsection{Circulation and Shell burning}

In a non-rotating star pressure and density are constant on equipotentials, i.e. they are constant on spheres and from the equation of state, say, in the case of the perfect gas one can conclude that $T / \mu$ is also constant on spheres.

Let us now consider nuclear shell burning. Above the shell is the fuel, below are the ashes of higher molecular weight. The transition from fuel to ashes is continuous and therefore there is a transition region where molecular weight increases inwards. We now consider a slow perturbation of the type as it is indicated in Figure 7. We discuss the conditions on a sphere $S$ which is right in the shell. Let $A$ be an area where material is moving outwards through this sphere. There, material enriched in ashes, with a higher molecular weight, passes through $S$, while in the region $B$ where matter is moving inwards, material enriched in fuel, with a lower molecular weight, penetrates the sphere. Now since $T / \mu$ is constant on $S$, the temperature in the area $A$ is higher while in area $B$ the temperature is lower. The effect of chemical composition diminishes the nuclear reactions in area $A$ and increases the nuclear reaction rate in area $B$, but the variation of temperature does exactly the opposite. The variation of chemical composition tries to damp the circulation while the effect of temperature tries to enhance the motion. In order to find out whether or not shell burning is stable against this type of perturbation, one has to do a more detailed stability analysis. The first attempt in this direction has been done by Mestel (1957), later by Kippenhahn (1967), and then by Rosenbluth and Bahcall (1973), and by Richstone (1973). As one would already expect from our heuristic consideration, the higher the temperature dependence of nuclear reactions, the more likely the shell is to be unstable. This indeed comes from the detailed study of the stability problem, but one also has to take into account the effects of radiative transfer.

Some stability criteria have been given by Kippenhahn (1967). Biermann (1968) investigated the stability of shell burning in Red Giants, with the result that the shells seem to be stable. Recently Richstone (1973) has studied stellar models near and during the onset of helium flash, and he found that all models investigated which were spherically stable were also stable with respect to non-spherical perturbations. Some flash models were unstable but the $e$-folding time of the non-spherical instability was longer than the $e$-folding time of the spherical instability. It is therefore doubtful whether this type of circulation can have any influence on the evolution of the stars. 


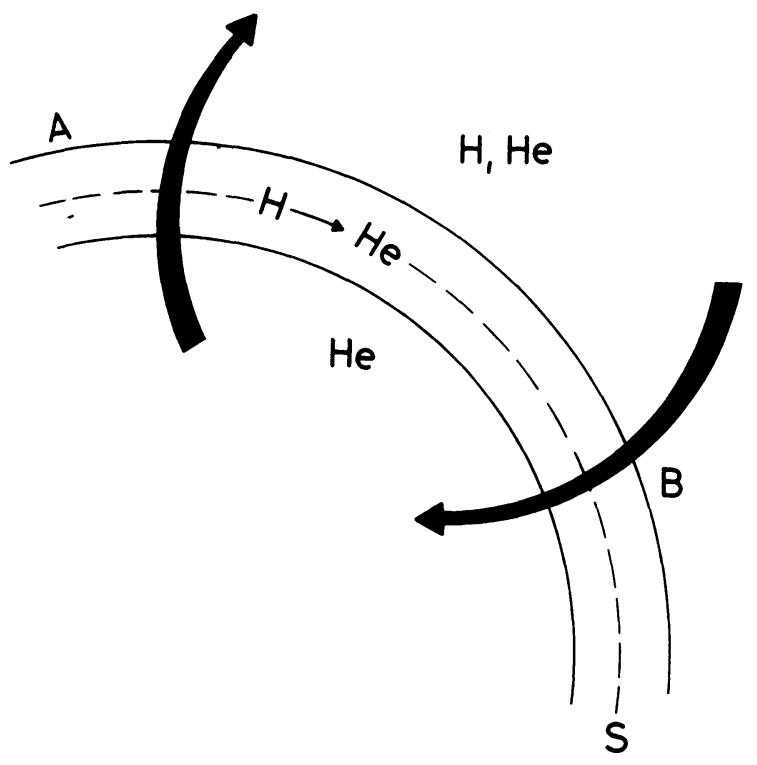

Fig. 7. In a spherically symmetric star with shell burning a non-spherical perturbation (black arrows) brings ashes into the shell in region $A$ and at the same time $T$ increases while in region $B$ new fuel is brought into the shell while the temperature decreases.

\subsection{Plume mixing}

Recently Scalo and Ulrich (1973) have postulated a new mixing process which might

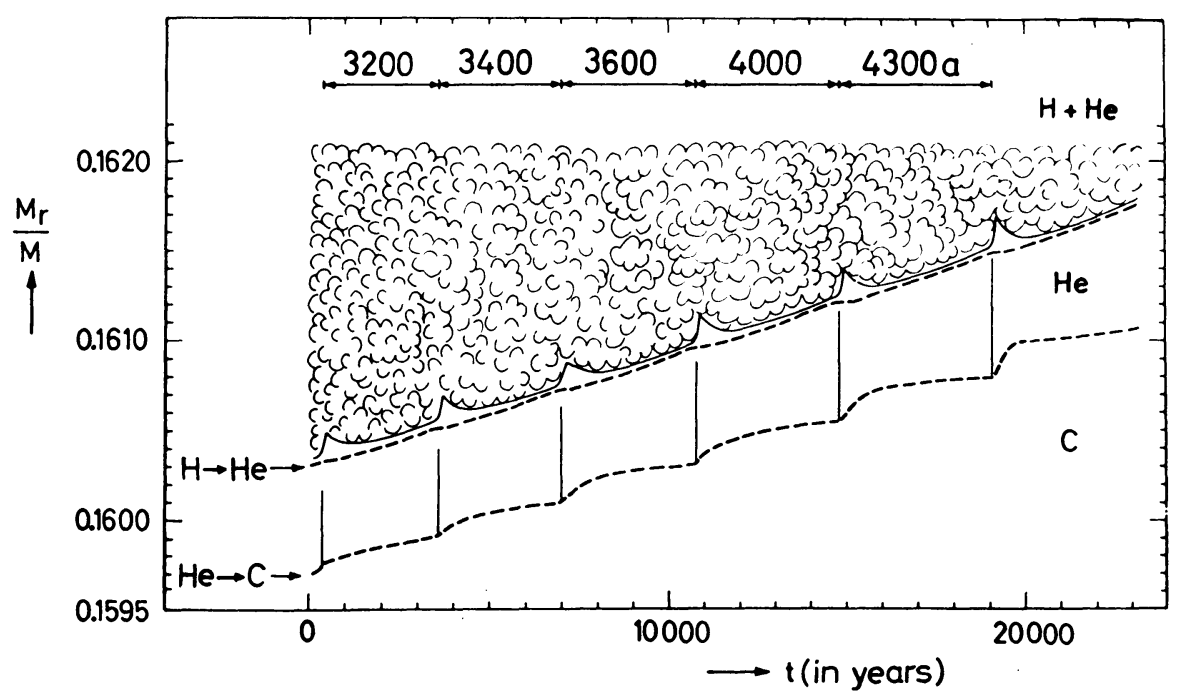

Fig. 8. Thermal pulses in a star of $5 M_{\odot}$ (Weigert, 1966). The cloudy region gives the outer convective zone. The broken lines indicate the two shell sources eating outward. In intervals of about 4000 years thermal pulses occur forming short-living convective zones (vertical lines above the helium burning shell) which approach the hydrogen burning shell. The Scalo-Ulrich plume mixing is supposed to occur as soon as the top of such a convective zone penetrates into the hydrogen burning shell. 
occur in an evolved star if carbon enriched material is brought up into a hydrogen rich outer region. This might be expected after many cycles of thermal pulses (Weigert, 1966) as indicated in Figure 8. During such a pulse a carbon enriched mixture is brought into the envelope by the convective zone which forms just above the helium burning shell during the pulse.

Due to the irregular convective pattern the material at the bottom of the envelope is heated in a non-spherically symmetric way, causing meridional circulation in the envelope. This motion would then bring hydrogen into the convective region where it would be mixed with the carbon enriched material (Figure 9). Since the CNOcycle is controlled by the $\mathrm{C}^{12}$ content the energy production due to the CNO-cycle would be strongly enhanced. Under happy circumstances this extra energy could be used to enhance the circulation. Scalo and Ulrich give a non-linear model for this type of motion and discuss the consequences of this type of mixing on chemical abundances. The results look very promising.

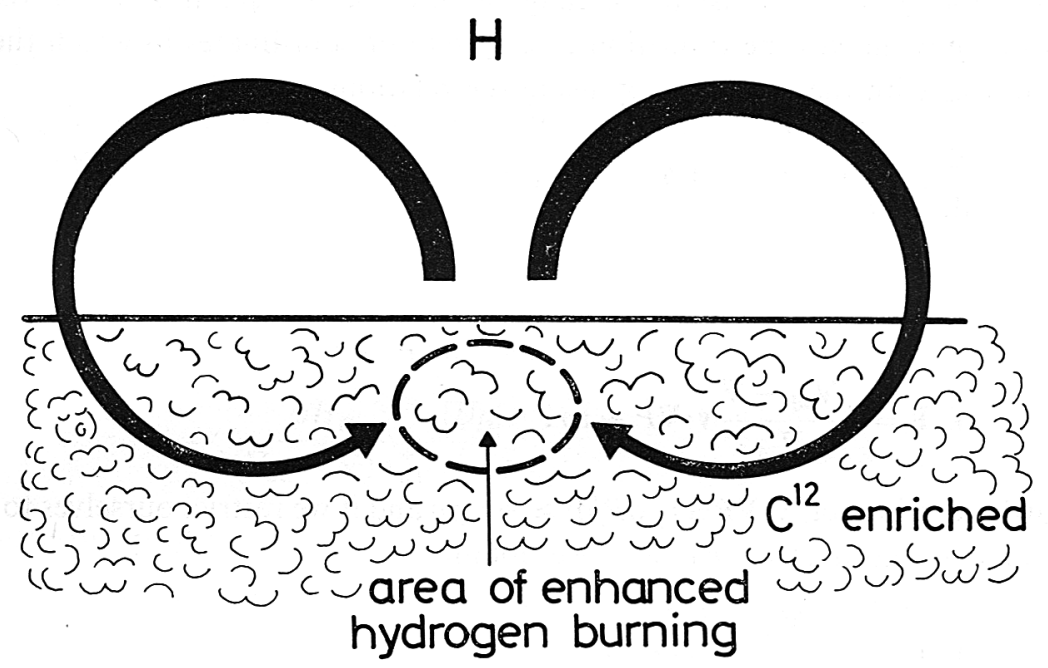

Fig. 9. Scalo-Ulrich plume mixing. Enhanced CNO burning in the convective, $\mathrm{C}^{12}$ enriched zone drives circulation.

One would like to see this model of plume mixing worked out in detail. Maybe one should try to learn more of the driving mechanism by a stability analysis of a purely spherically symmetric model. In such a model non-spherical perturbations similar to those discussed in the preceding section should be applied. But the difference between this and the former case would be that mixing occurs at a certain depth. Such an approach could possibly help to find whether the excess energy released by the CNO-cycle would really be fed into the circulation, driving the motion rather than damping it, which in principle is also possible.

On the other hand model calculations beyond the phases covered by Weigert are 
necessary in order to find whether the convective zone can really penetrate into the hydrogen burning shell.

\section{Acknowledgement}

Part of this work was financed by the Deutsche Forschungsgemeinschaft.

\section{Appendix}

\section{A1. Estimate FOR $v_{\text {EV }}$}

In a system of coordinates in which the matter of the star is at rest the first law of thermodynamics gives

$$
\nabla \cdot \mathbf{F}=\varrho \varepsilon-c_{P} \varrho \frac{\mathrm{d} T}{\mathrm{~d} t}+\delta \frac{\mathrm{d} P}{\mathrm{~d} t} .
$$

Here, $\mathbf{F}$ is the radiative flux and $\varepsilon$ the nuclear energy generation, while all the other symbols either have their usual meaning or have been explained in the foregoing text. If one now makes the transition to a system of coordinates in which the stellar matter moves with the velocity $\mathbf{v}$ by using the relations

$$
\begin{aligned}
& \begin{array}{l}
\mathrm{d} \\
\mathrm{d} t
\end{array}=\frac{\partial}{\partial t}+\mathrm{v} \cdot \nabla, \quad \nabla T=\frac{T}{P} \nabla \nabla P \\
& \nabla=\mathrm{d} \ln T / \mathrm{d} \ln P, \quad \nabla_{\mathrm{ad}}=(\mathrm{d} \ln T / \mathrm{d} \ln P)_{\mathrm{ad}}=\begin{array}{c}
P \delta \\
c_{P} \varrho T
\end{array}
\end{aligned}
$$

one obtains

$$
\boldsymbol{\nabla} \cdot \mathbf{F}=\delta \frac{\nabla_{\mathrm{ad}}-\nabla}{\nabla_{\mathrm{ad}}} \mathbf{v} \cdot \nabla P+\varepsilon \varrho .
$$

In a slowly rotating star $\mathbf{v} \cdot \nabla P \simeq v_{r} \mathrm{~d} P / \mathrm{d} r \approx-v_{r} g \varrho$ and if we restrict ourselves to regions where there are no nuclear reactions we obtain

$$
\nabla \cdot \mathbf{F}=-\delta \frac{\nabla_{\mathrm{ad}}-\nabla}{\nabla_{\mathrm{ad}}} g \varrho v_{r} .
$$

That in rotating stars there is normally no solution with $v_{r}=0$ comes from the fact that $\boldsymbol{\nabla} \cdot \mathbf{F}$ does not vanish.

As an estimate which is good for slowly rotating stars one can say (Baker and Kippenhahn, 1959, 144-145)

$$
\nabla \cdot \mathbf{F} \approx \frac{3 L_{r}}{4 \pi r^{3}} \chi_{r}
$$

and from Equation (A1.4) one obtains

$$
v_{\mathrm{EV}}=\left|v_{r}\right| \approx \frac{\nabla_{\mathrm{ad}}}{\delta\left(\nabla_{\mathrm{ad}}-\nabla\right)} \frac{L_{r} \chi_{r}}{M_{r}} 4 \pi r G \varrho .
$$


A2. $\mu$-Motion

If a blob of material in a star is carried with the velocity $v$ either upwards $(v>0)$ or downwards $(v<0)$, its temperature is determined by the equation

$$
\frac{\partial \theta}{\partial t}=\left(\nabla-\nabla_{\mathrm{ad}}\right) \frac{v}{H_{P}}-\frac{1}{\tau_{\mathrm{KH}}} \theta
$$

(Kippenhahn, 1969) where $\theta=D T / T$ and where the time $\tau_{\mathrm{KH}}^{*}$ of thermal adjustment is given by Equation (7). If the velocity does not change very much during the time $\tau_{\mathbf{K H}}^{*}$ then a quasi-steady state is achieved:

$$
\theta=-\left(\nabla_{\mathrm{ad}}-\nabla\right) \frac{v}{H_{P}} \tau_{\mathbf{K H}}^{*}
$$

For the case of different molecular weights inside and outside the blob, we obtain from $D P=0, D \varrho=0$

$$
-\delta_{T}^{D T}+\varphi \frac{D \mu}{\mu}=0,
$$

where $\delta=-(\partial \ln \varrho / \partial \ln T)_{P, \mu}, \varphi=(\partial \ln \varrho / \partial \ln \mu)_{P, T}(\delta, \varphi=1$ for an ideal gas $)$. Consequently, we have

$$
\theta=\frac{D T}{T}=\begin{aligned}
& \varphi D \mu \\
& \delta \mu
\end{aligned} .
$$

Combining Equation (A2.2) and (A2.4) yields

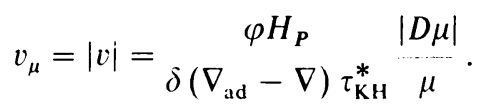

\section{A3. Magnetic Buoyancy}

In order to derive the formula for magnetic buoyancy we again start with Equation (A2.2). We consider the size of the blob to be small compared to $H_{P}$. We may therefore assume the gas pressure $P_{G i}$ in the blob and $P_{G e}$ in the surroundings as being constant over the blob's diameter. $P_{M}$ is a mean value of the magnetic pressure in the blob. We then define a dimensionless quantity $\zeta$ by

$$
P_{G i}=(1-\zeta) P, \quad P_{M}=\zeta P,
$$

where $P=P_{G i}+P_{M}=P_{G e}$ is the total pressure. We assume $\zeta$ to be small compared to unity.

$$
D P_{G}=P_{G i}-P_{G e}=-P_{M}=-\zeta P
$$

and from the equation of state

$$
\stackrel{D \varrho}{\varrho}=\alpha \underset{P_{G}}{D P_{G}}-\delta \frac{D T}{T}
$$


with $\alpha=(\partial \ln \varrho / \partial \ln P)_{T}(\alpha=1$ for an ideal gas). Since in hydrostatic equilibrium $D \varrho=0$ we conclude from Equation (A3.2), (A3.4)

$$
\theta=\frac{D T}{T}=-{ }_{\delta P_{G}}^{\alpha} P=-\begin{aligned}
& \alpha P_{M} \\
& \delta P_{G}
\end{aligned}=\begin{aligned}
& \alpha D P_{G} \\
& \delta P_{G}
\end{aligned} .
$$

And if we combine Equation (A2.2) and (A3.4) we find

$$
v_{M}=\frac{\alpha H_{P}}{\delta\left(\nabla_{\mathrm{ad}}-\nabla\right) \tau_{\mathrm{KH}}^{*}} P_{G} .
$$

\section{A4. Size OF THE FASTEST ELEMENTS}

We consider a spherical blob of diameter $d$ with $D \mu>0$. If we assume pressure equilibrium $(D P=0)$ and thermal adjustment $(D T=0)$ then $D \mu / \mu=D \varrho / \varrho \neq 0$ and a buoyancy force

$$
K=g V D \varrho={ }_{3}^{32 \pi} \operatorname{god}{ }^{3} \stackrel{D \mu}{\mu}
$$

acts on the blob where $V$ is its volume. It would then move with the Stokes velocity $v_{\mathrm{St}}$ defined by

$$
3 \pi f \eta v_{\mathrm{st}} d=K
$$

where $f$ is a dimensionless factor of order 1 , while $\eta$ is the viscosity. Thus, we obtain

$$
v_{\mathrm{St}}=\begin{array}{lll}
32 & g d^{2} & D \mu \\
9 f & v & \mu
\end{array},
$$

where $v$ is the kinematic viscosity $v=\eta / \varrho$. This was without thermal adjustment. With thermal adjustment without friction it would move with

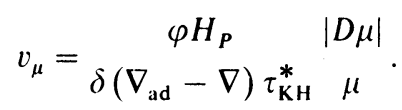

If we now express $v$ in units of the kinematic radiative viscosity $v_{R}$

$$
v_{R}=\frac{2 a T^{4}}{15 \frac{c \varkappa \varrho^{2}}{2}}
$$

and if we put $v_{\mathrm{St}}=v_{\mu}$, we obtain for the diameter of the blobs where friction becomes important

$$
d \approx\left(\frac{45 \nabla_{\mathrm{ad}} \varphi f}{8 \delta^{2}\left(\nabla_{\mathrm{ad}}-\nabla\right)} \cdot v_{R}\right)^{1 / 4} \sqrt{\frac{v_{R} c}{g_{r}}} .
$$

Ignoring the factor in front of the square root we obtain the estimate (12).

A5. A magnetic blob Lifting heAvier material

We assume a rising magnetic blob with $D \mu>0$. In this case 


$$
P_{M}>\frac{D \mu}{\mu}
$$

As the element rises along a certain path $P_{M} / P_{G i}$ changes to $\left(P_{M} / P_{G i}\right)+\mathrm{d}\left(P_{M} / P_{G i}\right)$ while $D \mu / \mu$ remains constant, if the element is moving through a chemically homogeneous region. Thus, if $\mathrm{d}\left(P_{M} / P_{G i}\right)>0$ then the effective buoyancy will become larger during the upward motion, while for $\mathrm{d}\left(P_{M} / P_{G i}\right)<0$ the upward motion will slow down. The variation of $P_{M} / P_{G i}$ during the upward motion is given by

$$
\mathrm{d}\left(\begin{array}{c}
P_{M} \\
P_{G i}
\end{array}\right)=\begin{aligned}
& \mathrm{d} P_{M} \\
& P_{G i}-P_{M} \mathrm{~d} P_{G i} \\
& P_{G i}
\end{aligned} .
$$

During the upward motion the density in the blob (and in the surroundings) changes according to

$$
\stackrel{\mathrm{d} \varrho}{\varrho}=\alpha \frac{\mathrm{d} P_{G i}}{P_{G i}}-\delta{ }_{T_{i}}^{\mathrm{d} T_{i}}=(\alpha-\nabla \delta) \frac{\mathrm{d} P_{G i}}{P_{G i}},
$$

where $\nabla=\mathrm{d} \ln T / \mathrm{d} \ln P$ gives the ratio of the relative variations of temperature to pressure in the surroundings. While the density is changing during the motion, the element expands and the frozen in magnetic field changes. We define a dimensionless quantity $\psi$ by

$$
\frac{\mathrm{d} P_{M}}{P_{M}}=\psi \stackrel{\mathrm{d} \varrho}{\varrho}
$$

and we obtain

$$
\mathrm{d}\left(\begin{array}{l}
P_{M} \\
P_{G i}
\end{array}\right)=[\dot{\psi}(\alpha-\nabla \delta)-1] \underset{P_{G i}}{\mathrm{~d} P_{G i}} \cdot P_{G} P_{G} .
$$

During an upward motion $\mathrm{d} P_{G i}<0$ and therefore

$$
\psi(\alpha-\nabla \delta) \leqslant 1
$$

is the condition that the rising element will not slow down. For an ideal gas $\alpha=\delta=1$, and we obtain from Equation (A5.6):

$$
\psi \leqslant \frac{1}{1-\nabla} \approx \frac{4}{3}
$$

where on the right we have taken $\nabla=0.25$, a value typical for radiative regions in stars.

The simplest case of a magnetic tangle would be a cylinder of radius $R$ with a magnetic field parallel to its axis. As it rises $\varrho \sim 1 / R^{2}, B \sim 1 / R^{2}$ and $P_{M} \sim 1 / R^{4}$ and therefore $P_{M} \sim Q^{2}$ or $\psi=2$ from which we can conclude that the upward motion will slow down. For a spherical tangle the magnetic pressure falls off with falling density even faster, $\psi$ is therefore even larger than 2 and the rising tangle is decelerated even faster. 


\section{References}

Aller, L. H.: 1961, The Abundance of the Elements, Interscience Publ. Inc., New York.

Baker, N. and Kippenhahn, R.: 1959, Z. Astrophys. 48, 140.

Biermann, P.: 1968, Diplomarbeit.

Demarque, P. and McClure, R. D.: 1973, Monthly Notices Roy. Astron. Soc. 164, 5.

Eddington, A. S.: 1925, Observatory 48, 73.

Finzi, A. and Wolf, R. A.: 1968, Astrophys. J. 153, 865.

Fricke, K. J.: 1968, Z. Astrophys. 68, 317.

Fricke, K. J. and Kippenhahn, R.: 1972, Ann. Rev. Astron. Astrophys. 10, 45.

Goldreich, P. and Schubert, G.: 1967, Astrophys. J. 150, 571.

Gurm, H. S. and Wentzel, D. G.: 1967, Astrophys. J. 149, 139.

Kippenhahn, R.: 1963, in L. Gratton (ed.), Star Evolution, Acad. Press. New York, p. 330.

Kippenhahn, R.: 1967, Z. Astrophys. 67, 271.

Kippenhahn, R.: 1969, Astron. Astrophys, 2, 309.

Kippenhahn, R. and Möllenhoff, C.: 1974, Astrophys. Space Sci., in press.

Köhler, H.: 1973, Astron. Astrophys. 25, 467.

Krause, F. and Rädler, K. H.: 1971, in Ergebnisse der Plasmaphysik und der Gaselektronik, Band II,

R. Rompe and M. Steenbeck, Berlin, p. 3.

Mestel, L.: 1953, Monthly Notices Roy. Astron. Soc. 113, 716.

Mestel, L.: 1957, Astrophy's. J. 126, 550.

Paczyński, B.: 1973, preprint.

Parker, E. N.: 1970, Ann. Rev. Astron. Astrophyis. 8, 1.

Richstone, D. O.: 1973, preprint.

Rosenbluth, M. N. and Bahcall, J. N.: 1973, Astrophys. J. 184, 9.

Sackmann, I.-J. and Weidemann, V.: 1972, Astrophys. J. 178, 427.

Scalo, J. M. and Ulrich, R. K.: 1973, Astrophys. J. 183, 151.

Sweet, P. A., 1950, Monthly Notices Roy. Astron. Soc. 110, 548.

Thomas, H.-C.: 1967, Z. Astrophy's. 67, 420.

Vogt, H.: 1925, Astron. Nachr. 223, 229.

Weigert, A.: 1966, Z. Astrophys. 64, 395. 